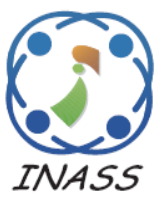

\title{
Microaneurysms Detection Using Grey Wolf Optimizer and Modified K-Nearest Neighbor for Early Diagnosis of Diabetic Retinopathy
}

\author{
Manohar Pundikal ${ }^{1 *}$ \\ Mallikarjun Sayabanna Holi ${ }^{2}$ \\ ${ }^{1}$ RNS Institute of Technology, India \\ ${ }^{2}$ University BDT College of Engineering, Davangere (A Constituent College of VTU, Belagavi), India \\ * Corresponding author’s Email: manoharasp@gmail.com
}

\begin{abstract}
In recent decades, Diabetic Retinopathy (DR) is a progressive eye disease that causes severe eye injuries if it is not detected and treated on time. Accurate microaneurysms detection is a vital step for early detection of DR, because it is the primary sign of disease. In this paper, a six-phase model is introduced for detecting microaneurysms from the fundus retinal images for early diagnosis of DR. Initially, lower light retinal image enhancement, image normalization, gradient weighting and shade correction are applied for improving the visibility level of fundus retinal images, which are acquired from the e-ophtha, and DiaRetDB1 datasets. Further, the hessian-based filter, and Otsu thresholding with the morphological operator are employed to eliminate blood vessel regions from the microaneurysms regions. Next, a grey wolf optimizer is used for predicting the correctness of the segmented microaneurysms regions. After segmentation, feature extraction: shape and Gray Level Co-occurrence Matrix (GLCM) features and classification: Modified K Nearest Neighbor (MKNN) are used to extract features from microaneurysms regions and to classify microaneurysms and non-microaneurysms regions. The simulation result showed that the proposed model achieved effective performance in microaneurysms detection compared to the existing models such as H-maximamultilevel thresholding-multilayer perceptron and statistical geometrical features. The proposed model achieved 99.10\% and $99.90 \%$ of accuracy on e-ophtha and DiaRetDB1 datasets, which are effective related to the existing models in microaneurysms detection.
\end{abstract}

Keywords: Grey wolf optimizer, Hessian based filter, Modified K-nearest neighbor, Microaneurysms detection, Otsu thresholding with a morphological operator.

\section{Introduction}

Currently, DR is the most frequent cause of new blindness cases for age groups between 20 and 70 years. DR is an eye complication that is caused due to diabetes mellitus, which further result in cardiovascular disease, diabetic neuropathy, diabetic nephropathy, and stroke [1,2]. Diabetes mellitus is an epidemic, due to modern lifestyles (urbanization), longer lifespan, and social and environmental factors like lack of physical activity, diet and obesity [3, 4]. The screening is the best way for early detection of DR, where fundus image is commonly used for the assessment and detection of ocular diseases [5, 6]. Generally, retinal fundus images contain pathological features (exudates, and microaneurysms) and normal features (fovea, blood vessels, and optic disc) [7].
Among pathological features, microaneurysms are the 1st sign of the presence of proliferative DR. Recently, many research works are developed for recognizing microaneurysms from the fundus retinal images. The methodologies used for microaneurysms recognition are divided into 3 types like template matching-based methodologies, supervised classification and morphological image processing [8-10]. The most common problem in the existing methods is that the features for non- microaneurysms vary in a wide range, so the large training set consumes more processing time and leads to class imbalance problems. In this paper, an attempt has been done for detecting the microaneurysms regions from the fundus retinal images for early recognition of DR. The major contributions of this paper are listed below; 
- Firstly, the fundus retinal images are acquired from the e-ophtha and DiaRetDB1 datasets, where the image denoising is accomplished by utilizing lower light retinal image enhancement, image normalization, gradient weighting, and shade correction. The image normalization and low light retinal image enhancement techniques are used to maintain the pixel values that significantly improves the visibility level of the collected retinal images.

- Next, the pixel intensity variations in the green plane image background are eliminated from the enhanced images, where the resultant image is the "shade corrected" image. Though, the shade corrected image is obtained by subtracting the background image from the green image.

- After pre-processing the images, blood vessel removal is accomplished by utilizing Otsu thresholding with a morphological operator and Hessian-based filter, where these methods segment and separate the overlapped microaneurysms and non-microaneurysms regions.

- Then, Grey Wolf Optimizer (GWO) is applied for predicting the correctness of segmented microaneurysms regions. After segmenting the microaneurysms regions, shape features and GLCM features are used for extracting the feature vectors from the microaneurysms regions and then MKNN classifier is applied for classifying both microaneurysms and nonmicroaneurysms regions. In the experimental phase, the GWO-MKNN model is investigated in light of accuracy, sensitivity, specificity, error rate, False Omission Rate (FOR), and miss rate.

This paper is structured as follows. In Section 2, some papers on the topic "microaneurysms detection" are reviewed. In Section 3, the GWO-MKNN model is briefly explained and experimental evaluation of GWO-MKNN model is discussed in Section 4. Conclusion of this research study is clearly stated in Section 5.

\section{Literature survey}

Mazlan [11] developed an automated framework for microaneurysms detection in retinal images. After collecting the images from the e-ophtha dataset, contrast enhancement was applied to improve the visibility of the images. Next, H-maxima and thresholding approaches were utilized to reduce the image intensity level to segment the microaneurysms regions. A total of fifteen features (color, shape, statistical moments, size, etc.) were employed to extract the features from the segmented regions. Then, the obtained feature values were fed to Support Vector Machine (SVM) and Multilayer Perceptron (MLP) to classify the images into three classes like background, blood vessels and microaneurysms. Experimental results showed that the presented framework obtained good performance in microaneurysms detection related to existing frameworks using specificity, accuracy, and sensitivity. The dimensionality issues will occur by utilizing more features that increase the complexity of the framework. Additionally, Veiga [12] utilized laws texture features to detect the microaneurysms from the e-ophtha dataset. In this literature study, the SVM classifier performs both object and pixel-based classification to diminish the false detections to classify the microaneurysms regions. Still, some false positives appear over thin vessels that degrades the performance of microaneurysms detection.

Akram [13] presented a three-phase model for early recognition of microaneurysms. In the first phase, filter banks, morphological operations and contrast normalization were applied to improve the contrast and to segment the microaneurysms regions from the images. In the second phase, color features, grey level features, shape-based features and statistical features were utilized for extracting feature vectors from the segmented regions. In the final stage, a hybrid classifier; SVM with Gaussian mixture model was used to classify the non-microaneurysms and microaneurysms regions. Experimental results showed that the presented model attained effective performance in microaneurysms detection compared to the prior models in terms of specificity, positive predictive value, accuracy and sensitivity. In image processing applications, the SVM classifier is not appropriate for large datasets and also it does not perform well when the dataset has noise (overlapping of classes). Derwin [14] developed a framework for automatic microaneurysms detection. Initially, normalization, contrast enhancement and luminosity correction were done to improve the quality of retinal images. Next, a morphological closing operation was applied to segment the optical disc and microaneurysms regions. Further, a local neighborhood differential coherence pattern was used to extract the feature vectors and then Feed Forward Neural Network (FFNN) was employed to classify the non-microaneurysms and microaneurysms regions. This extensive experiment showed that the developed framework performed well in microaneurysms detection. The developed FFNN is a single-layer perceptron that only learns linearly separable patterns, which is not adaptable for multiclass classification. 
Long [15] developed an improved enhancement function based on the eigenvalues of the Hessian matrix for retinal blood vessel and microaneurysms segmentation. Using shape property and connected component analysis, the blood vessels were removed from the microaneurysms regions. Next, the directional local contrast features were utilized for extracting feature vectors from the segmented microaneurysms regions. Finally, the deep learning classifier was used to classify the microaneurysms and non-microaneurysms regions. The major concerns in deep learning classifier are long training time and require higher computational resources (best graphics processing unit). Manjaramkar, and Kokare, [16] presented a model to segment and detect the microaneurysms in color fundus retinal images. In this study, a set of features were developed based on the statistics of geometrical properties of connected regions that discriminate both non-lesion and lesion pixels. In this work, the presented model performance was evaluated on the DiaRetDB1 dataset using accuracy, sensitivity and specificity. Both noise problems and non-uniform illumination are ineffectively tackled by the developed model that degrades the classification performance.

Putra [17] used morphology contrast enhancement technique, homomorphic method and contrast limited adaptive histogram equalization technique for image resizing and cropping. The pre-processed image was given as the input to deep learning techniques such as ResNet101, GoogLeNet, ResNet50 and ResNet18 for feature extraction. Then, feature selection was carried out by reliefF and principle component analysis, and then classification was performed using naïve Bayes classifier with SVM. Qomariah [18] developed a new deep learning framework which modifies UNet using residual unit with modified identity mapping in order to perform microaneurysm segmentation. Here, the identity map modification using convolutional layers and batch normalization were used to extract feature vectors and then the Residuals in UNet was employed for feature degradation and classification. As seen in the resulting phase, the computational complexity of the developed models was comparably high related to the existing deep learning techniques. To highlight the above-stated concerns, the GWO-MKNN model was proposed to improve microaneurysms detection performance.

\section{Methodology}

In microaneurysms detection, the proposed GWOMKNN model includes six phases; image collection: e-ophtha, and DiaRetDB1, image pre-processing:

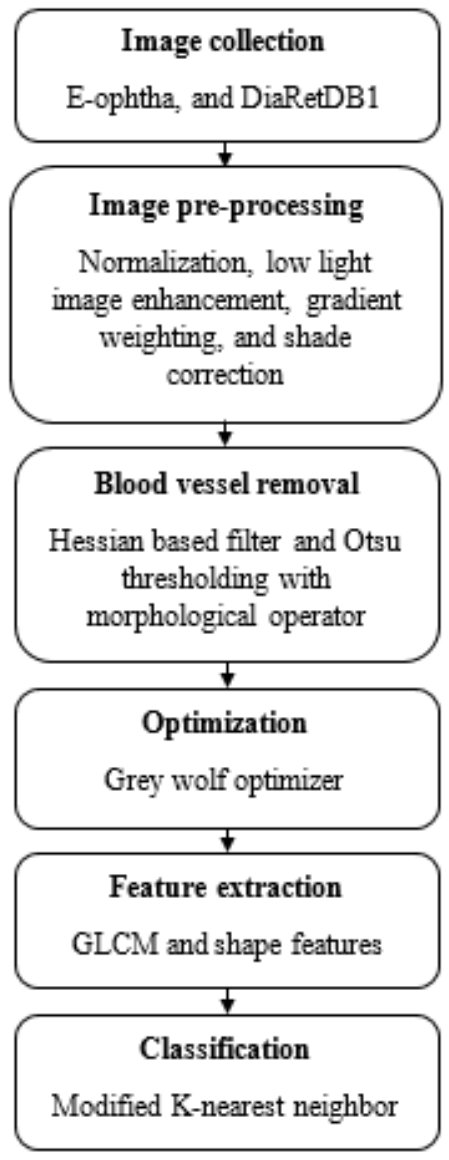

Figure. 1 Work flow of proposed GWO-MKNN model

normalization, gradient weighting, low light image enhancement, and shade correction, blood vessel removal: Otsu thresholding with morphological operator and hessian based filter, optimization: GWO, feature extraction: GLCM and shape features, and classification: MKNN. The workflow of the proposed GWO-MKNN model is graphically depicted in Fig. 1.

\subsection{Image acquisition and pre-processing}

In this research study, e-ophtha and DiaRetDB1 datasets are used to analyse the proposed GWOMKNN model performance. The e-ophtha dataset includes 2 sub-datasets such as e-ophtha microaneurysms and e-ophtha exudates. Here, eophtha microaneurysms dataset is undertaken for microaneurysms detection that contains 148 microaneurysms images and 233 normal images [19]. In e-ophtha dataset, the fundus retinal images are collected from 4 image resolutions, which are ranges from $1440 \times 960$ to $2544 \times 1696$ pixels with $45^{0}$ field view. Additionally, the DiaRetDB1 dataset comprises 89 color fundus retinal images in that 5 images belong to normal signs and the remaining 84 fundus images belong to microaneurysms patients. In 


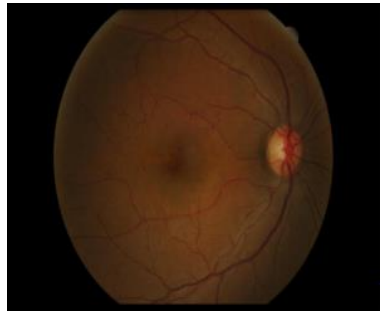

(a)

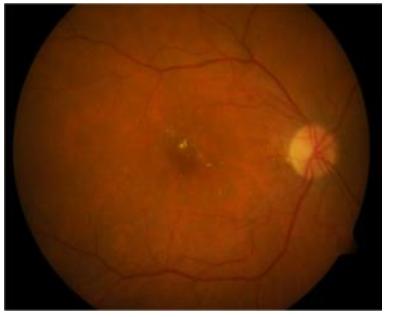

(b)
Figure. 2 Sample collected fundus retinal images: (a) eophtha dataset and (b) DiaRetDB1 dataset

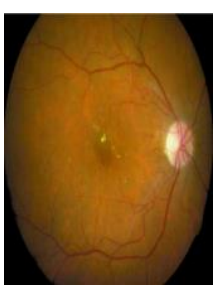

(a)

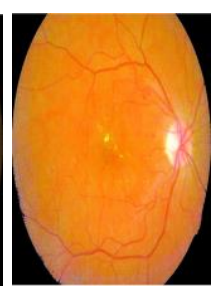

(b)

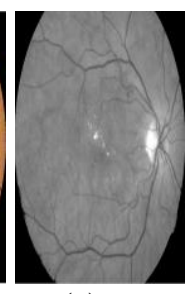

(c)

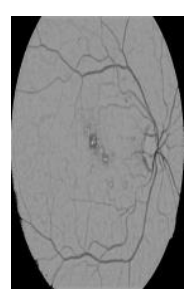

(d)
Figure. 3 Pre-processed images: (a) normalized image, (b) low light enhancement image, (c) gradient weighting image and (d) shade corrected image

this dataset, the images are captured using $50^{\circ}$ field view camera by varying the image settings [20]. The sample collected images of e-ophtha and DiaRetDB1 datasets are graphically depicted in Fig. 2.

After collecting the fundus retinal images from eophtha and DiaRetDB1 datasets, image normalization is used to vary the pixel intensity values of the images which help to improve the visibility level of the collected images. Image normalization is mathematically represented in the Eq. (1), where $I$ is indicated as original fundus retinal images, $I N$ is denoted as normalized fundus retinal images, and Min and Max is represented as minimum and maximum pixel intensity value that ranges from 0 to 255 .

$$
I N=(I-\text { Min }) \times \frac{\text { newMax-newMin }}{\text { Max-Min }}+\text { newMin }
$$

Then, gradient weighting and low light enhancement techniques are applied to improve the contrast and brightness of the normalized image $I N$, where these techniques help to extract the hidden details [21]. In shade correction, the enhanced image $I E$ is filtered $($ mask $=35 \times 35)$ to obtain the background image to correct the non-uniform illumination of the green channel retinal fundus image [22]. Then, the background image is subtracted from the filtered image, where the resultant image is the dark image "shade corrected image $I D$ ", so it is easy to view any structures. The sample preprocessed image is graphically depicted in Fig. 3.

\subsection{Blood vessel removal}

After pre-processing the fundus retinal images, shade corrected image $I D$ is fed to a hessian-based filter and Otsu thresholding with a morphological operator to remove blood vessels from the microaneurysms regions. The hessian-based filter works based on the Hessian matrix, where the eigenvalues and eigenvectors of the hessian matrix are related to vascular intensity and direction. In shade corrected image $I D$, the hessian matrix $(2 \times 2$ matrix) is calculated using 2 nd order derivative which is mathematically defined in Eq. (2).

$$
H=\left[\begin{array}{ll}
\frac{\partial^{2} I D}{\partial x^{2}} & \frac{\partial^{2} I D}{\partial x \partial y} \\
\frac{\partial^{2} I D}{\partial y \partial x} & \frac{\partial^{2} I D}{\partial y^{2}}
\end{array}\right]
$$

Here, the eigenvalues of $H$ is represented as $\lambda_{1}$ and $\lambda_{2}$ and eigenvectors of $H$ is denoted as $e_{1}$ and $e_{2}$. The 2 nd order derivative of shade corrected retinal image $I D$ at a point $(x, y)$ is defined as a convolution with Gaussian filter derivative $G$ at scale $\rho$ that is defined in the Eqs. (3) and (4).

$$
H(x, \rho)=\rho^{2} I D(x) \times \frac{\partial^{2}}{\partial x_{i} \partial x_{j}} G(x, \rho), i, j=1,2
$$

Where,

$$
G(x, \rho)=\frac{1}{2 \pi \rho^{2}} \exp \left(-\frac{x^{2}+y^{2}}{2 \rho^{2}}\right)
$$

Where, $G(x, \rho)$ is represented as Gaussian convolution kernel, $\left|\lambda_{1}\right| \leq\left|\lambda_{2}\right|$ is denoted as elongated vascular structures and $\lambda_{2}<0$ is represented as bright vascular structures on a dark background. The enhancement of fundus retinal image vascular structure is performed based on the analysis of magnitudes and signs of Eigenvalues. The enhancement filter function $\vartheta$ at a particular scale $\rho$ is mathematically defined in Eq. (5).

$$
\vartheta(\rho)=\begin{array}{cc}
0 & \lambda_{2}>0 \\
1-\exp \left(\frac{-S^{2}}{2 \kappa^{2}}\right) & \text { otherwise }
\end{array}
$$

Where $S=\sqrt{\lambda_{1}^{2}+\lambda_{2}^{2}}$ denoted as structure-ness of the image, and $\kappa$ controls the sensitivity of S. The filter response of $F(x)$ at dissimilar scales $\rho$ is obtained by maximizing the filter function $\vartheta$, which is mentioned in Eq. (6). 


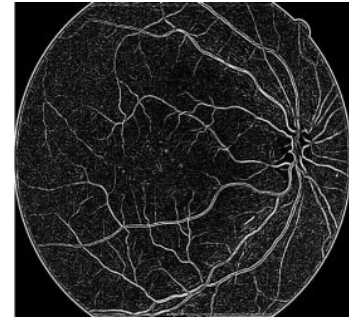

(a)

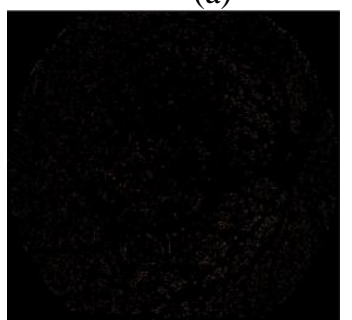

(c)

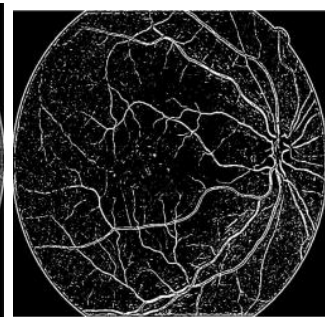

(b)

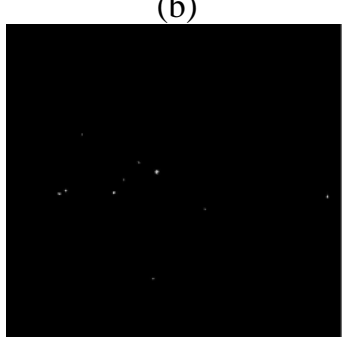

(d)
Figure. 4 Segmented images: (a) Hessian based filter, (b) Otsu thresholding, (c) morphological operator, and (d) GWO algorithm

$$
F(x)=\max \{\vartheta[\operatorname{Eigen}(H(x, \rho))]\}, \rho_{\min } \leq \rho \leq
$$

The output of Hessian based filter $F(x)$ is given as the input to Otsu thresholding approach to identify the maximum separability of microaneurysms and non-microaneurysms regions. The Otsu thresholding approach divides the filtered image $F(x)$ into two regions such as light $F_{0}$ and dark region $F_{1}$ that are mathematically defined in the Eqs. (7) and (8).

$$
\begin{aligned}
& F_{0}=\{0,1, \ldots, t\} \\
& F_{1}=\{t, t+1, \ldots ., l-1, l\}
\end{aligned}
$$

Where, $t$ is stated as a threshold value. By reducing the weighted group variance 1 , optimal threshold value $t$ is identified, where the weight is represented by the probability of individual groups $p(i)$ that is determined by using Eq. (9).

$$
p(i)=\frac{\text { number }\{(r, c) \mid \operatorname{image}(r, c)=i\}}{(R, C)}
$$

Where, $c$ is represented as a column of fundus retinal image, $r$ is indicated as a row of fundus retinal image, $R$ and $C$ are stated as a number of rows and columns of fundus retinal images. The weight $w_{b}(t)$, mean $\mu_{b}(t)$, and variance $\sigma_{b}^{2}(t)$ of the class $F_{0}$ is calculated using Eq. (10).

$$
\begin{aligned}
w_{b}(t) & =\sum_{i=1}^{t} p(i), \mu_{b}(t)=\frac{\sum_{i=1}^{t} i \times p(i)}{w_{b}(t)}, \\
\sigma_{b}^{2}(t) & =\frac{\sum_{i=1}^{t}\left(i-\mu_{b}(t)\right)^{2} \times p(i)}{w_{b}(t)}
\end{aligned}
$$

Similarly, weight $w_{f}(t)$, mean $\mu_{f}(t)$, and variance $\sigma_{f}^{2}(t)$ of the class $F_{1}$ is computed using the Eq. (11).

$$
\begin{aligned}
& w_{f}(t)=\sum_{i=t+1}^{l} p(i), \mu_{f}(t)= \\
& \frac{\sum_{i=t+1}^{l} i \times p(i)}{w_{f}(t)}, \sigma_{f}^{2}(t)=\frac{\sum_{i=t+1}^{l}\left(i-\mu_{f}(t)\right)^{2} \times p(i)}{w_{f}(t)}
\end{aligned}
$$

Finally, the optimal threshold value $t$ is achieved with minimum within-class variance $\sigma_{w}^{2}$ as indicated in Eq. (12).

$$
\sigma_{w}^{2}=w_{b}(t) \times \sigma_{b}^{2}(t)+w_{f}(t) \times \sigma_{f}^{2}(t)
$$

Then, the morphological transforms related to blood vessel size and shape distribution are applied to re-size the shape and size of microaneurysms and non microaneurysms regions. The resized retinal fundus images are fed to the GWO algorithm to predict the correctness of the segmented microaneurysms regions. Generally, GWO algorithm work based on three steps like en-circling, hunting, and attacking the prey. Assume, 3 best solutions $\alpha, \beta$, and $\delta$ to model the hierarchy of wolves. Initially, the grey wolves are en-circled utilizing the Eqs. (13) and (14).

$$
\begin{aligned}
& D=\left|M \cdot X_{\text {prey }}(T)-X_{\text {wolf }}(T)\right| \\
& X_{\text {wolf }}(T+1)=X_{\text {prey }}(T)-A . D
\end{aligned}
$$

Where, $X_{\text {wolf }}$ is represented as grey wolf position, $X_{\text {prey }}$ is indicated as prey position, $T$ is stated as current iteration, $A$ and $M$ are stated as coefficient vectors that are computed using the Eqs. (15) and (16).

$$
\begin{aligned}
& A=2 \vec{a} \cdot \vec{r}_{1}-\vec{a} \\
& M=2 \vec{r}_{2}
\end{aligned}
$$

Where, $\vec{a}$ is minimized from 2 to 0 throughout iterations and $\vec{r}_{1}$ and $\vec{r}_{2}$ are stated as random values that range between 0 to 1 . The 3 best solutions of $\alpha, \beta$, and $\delta$ are achieved to imitate the hunting behavior of grey wolves. The residual search agent: omega updates the location based on Eqs. (17), (18) and (19). The vessel enhanced and segmented images are stated in Fig. 4.

$$
\begin{gathered}
D_{\alpha}=\left|M_{1} \cdot X_{\alpha}-X\right|, D_{\beta}=\left|M_{2} \cdot X_{\beta}-X\right|, D_{\delta}= \\
\left|M_{3} \cdot X_{\delta}-X\right|
\end{gathered}
$$




$$
\begin{aligned}
X_{1}=X_{\alpha}- & A_{1} \cdot D_{\alpha}, X_{2}=X_{\beta}-A_{2} \cdot D_{\beta}, X_{3}= \\
& X_{\delta}-A_{3} \cdot D_{\delta} \\
X(T+1)= & \frac{\vec{X}_{1}+\vec{X}_{2}+\vec{X}_{3}}{3}
\end{aligned}
$$

\subsection{Feature extraction and classification}

After the segmentation of microaneurysms and non microaneurysms regions, feature extraction is accomplished using GLCM features and shape features. GLCM and shape features are high-level descriptors, which are used to extract the features from microaneurysms and non microaneurysms regions to differentiate microaneurysms region from other regions. By using the high-level feature descriptors, the semantic space between the extracted feature subsets are reduced which leads to better disease classification. In this research, 3 shape features (perimeter, eccentricity, and orientation) and 21 GLCM features (autocorrelation, homogeneity, cluster prominence, energy, difference entropy, sum of squares, cluster shade, sum variance, contrast, maximum probability, correlation, inverse difference, variance, dissimilarity, entropy, inverse difference normalized, information measure of correlation, difference variance, inverse difference moment normalized, sum entropy and sum average) are employed to extract the feature vectors [23].

Then, the extracted feature vectors are fed to the MKNN to classify both microaneurysms and non microaneurysms candidate. In the conventional KNN classifier, the testing and training process completely depends on the $\mathrm{k}$ nearest neighbor. Recently, many distance measures are used to compute the distance between testing and training samples [24]. In that, Euclidean distance is used in the KNN classifier to calculate the distance between training and testing samples. The formula of Euclidean distance measure is mathematically defined in Eq. (20).

$$
\text { Euclidean distance }=\sqrt{\sum_{i=1}^{N}\left(q_{i}-p_{i}\right)^{2}}
$$

Where $\mathrm{N}$ is represented as a number of samples, $p_{i}$ and $q_{i}$ are stated as training and testing samples. The MKNN is inspired from the conventional KNN classifier, where the main objective of MKNN is to classify the images based on the most frequency tags in the set of neighbor tags. The MKNN classifier is also named as weighted KNN, where the query label is approximated by weighting the neighbors of the query. This process calculates the frequencies of the similar labelled neighbors to the total number of neighbors. The steps involved in MKNN are listed below.

- Compute the number of nearest neighbors $(\mathrm{k}$ value).

- Compute the distance between testing and training samples.

- Sort the distance and identify the nearest neighbors based on $k^{\text {th }}$ minimum distance and by weighting the neighbors of the query.

- $\quad$ Sort the categories of nearest neighbors.

- Utilize the simple majority of nearest neighbor's categories as the prediction value of new data.

\section{Experimental results and discussion}

The proposed GWO-MKNN model was simulated by MATLAB (2019a) environment on a computer with processor: Intel i7 core, operating system: windows 10, hard disk: $4 \mathrm{~TB}$, and RAM: 16 GB. The proposed GWO-MKNN model performance was compared with the existing models such as $\mathrm{H}$ maxima-multilevel thresholding-MLP [11], statistical geometrical features [16], and Modified Residuals UNet (MResUNet) [18] to validate its efficiency. In this work, the proposed GWO-MKNN model performance was investigated in light of accuracy, sensitivity, specificity, error rate, FOR and miss rate that are mathematically defined in the Eqs. (21-26).

$$
\begin{array}{r}
\text { Accuracy }=\frac{T P+T N}{T P+T N+F P+F N} \times 100 \\
\text { Sensitivity }=\frac{T P}{T P+F N} \times 100 \\
\text { Specificity }=\frac{T N}{T N+F P} \times 100 \\
\text { Error rate }=100-\text { accuracy } \\
\text { FOR }=\frac{F N}{F N+T N} \times 100 \\
\text { Miss rate }=\frac{F N}{F N+T P} \times 100
\end{array}
$$

Where, $T N$ is represented as true negatives, $T P$ is stated as true positives, $F N$ is represented as false negatives and $F P$ is stated as false positives.

\subsection{Quantitative performance on e-ophtha dataset}

In this section, e-ophtha dataset is used to validate the proposed GWO-MKNN model performance in light of accuracy, sensitivity, specificity, error-rate, 
Table 1. Performance evaluation of GWO-MKNN model on e-ophtha dataset in light of specificity, accuracy, and sensitivity

\begin{tabular}{|c|l|l|l|l|}
\hline \multicolumn{5}{|c|}{ e-ophtha dataset } \\
\hline \multirow{2}{*}{ Optimization } & \multicolumn{1}{|c|}{ Classifier } & \multicolumn{1}{c|}{ Accuracy (\%) } & \multicolumn{1}{c|}{ Sensitivity (\%) } & \multicolumn{1}{c|}{ Specificity (\%) } \\
\hline \multirow{3}{*}{ Without GWO } & Decision tree & 92.99 & 94.21 & 87.99 \\
\cline { 2 - 6 } & Neural network & 92 & 92.13 & 85 \\
\cline { 2 - 6 } & Random forest & 94.95 & 91.42 & 91.24 \\
\cline { 2 - 6 } & MKNN & 97 & 98.82 & 98 \\
\hline \multirow{3}{*}{ With GWO } & Decision tree & 96.43 & 94.75 & 97.12 \\
\cline { 2 - 6 } & Neural network & 74.32 & 98.10 & 73.67 \\
\cline { 2 - 5 } & Random forest & 98.46 & 65.23 & 98.34 \\
\cline { 2 - 5 } & MKNN & $\mathbf{9 9 . 1 0}$ & $\mathbf{9 8 . 9 2}$ & $\mathbf{9 9 . 0 1}$ \\
\hline
\end{tabular}

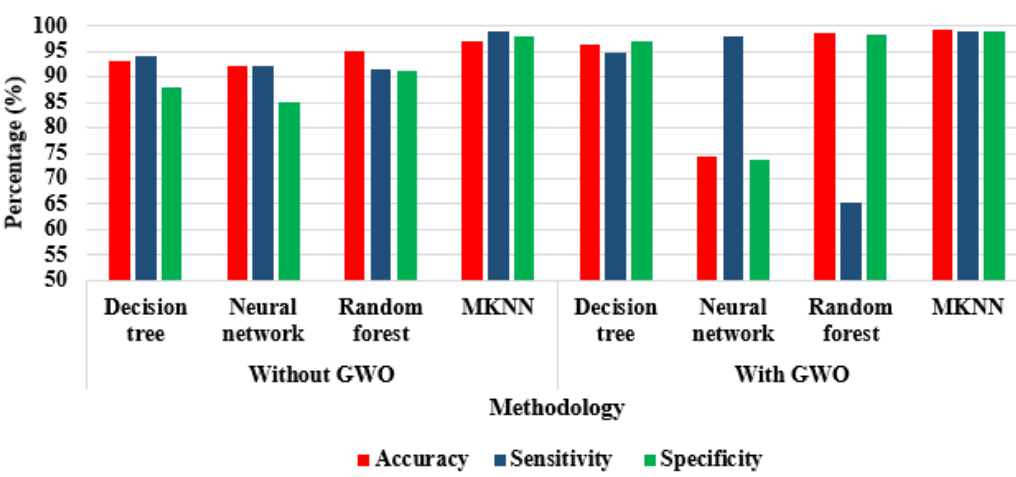

Figure. 5 Graphical comparisons of GWO-MKNN model on e-ophtha dataset in terms of sensitivity, specificity and accuracy

FOR, and miss-rate. The e-ophtha dataset consists of 233 images with no lesion and 148 images with microaneurysms in that $80 \%$ of images are used for training and $20 \%$ of images are used for testing. In this section, the proposed GWO-MKNN model is investigated with dissimilar existing classification techniques (decision tree, neural network, and random forest) and without using GWO. By inspecting table 1, the proposed GWO-MKNN model achieved a maximum classification accuracy of $99.10 \%$, sensitivity of $98.92 \%$, and specificity of $99.01 \%$. The graphical depiction of the GWOMKNN model on e-ophtha dataset in light of specificity, accuracy, and sensitivity is represented in Fig. 5.

Correspondingly in Table 2, the proposed GWOMKNN model is investigated in terms of error-rate, miss-rate and FOR. By inspecting Table 2, the proposed GWO-MKNN model attained minimum error rate of $0.90 \%$, miss rate of $3.74 \%$, and FOR of $0.02 \%$, which are better related to the existing classifiers (decision tree, neural network, and random forest) and without using GWO. Hence, the random forest and decision tree classifiers consume more time for training the data, because these classifiers generate a lot of trees and decides based on vote majority. The collected fundus retinal images are linear data, where the neural network is a non-linear classifier, so it showed limited performance in microaneurysms recognition. The graphical comparison of the GWO-MKNN model on e-ophtha dataset in terms of miss-rate, error-rate, and FOR is indicated in Fig. 6.

\subsection{Quantitative performance on DiaRetDB1 dataset}

Here, DiaRetDB1 dataset is used to validate the proposed GWO-MKNN model performance. The DiaRetDB1 dataset consists of 89 retinal fundus images (5 normal images and 84 microaneurysms images) in that $80 \%$ of the images are used for model training and $20 \%$ of the images are used for model testing. By inspecting Table 3, the proposed GWOMKNN model achieved a maximum accuracy of $99.90 \%$, a sensitivity of $99.03 \%$, and specificity of $99.21 \%$, which are better when compared to the existing classifiers (decision tree, neural network, and random forest) and without using GWO. The MKNN classifier without GWO achieved $98.95 \%$ of classification accuracy, where the proposed GWOMKNN model almost showed a $0.95 \%$ improvement 
Table 2. Performance evaluation of GWO-MKNN model on e-ophtha dataset in terms of miss-rate, error-rate, and FOR

\begin{tabular}{|c|c|c|c|c|}
\hline \multicolumn{5}{|c|}{ e-ophtha dataset } \\
\hline \multirow{3}{*}{ Optimization } & Classifier & Error rate (\%) & Miss rate (\%) & FOR (\%) \\
\hline \multirow{5}{*}{ Without GWO } & Decision tree & 7.01 & 10.28 & 0.21 \\
\cline { 2 - 5 } & Neural network & 8 & 11.27 & 0.05 \\
\cline { 2 - 5 } & Random forest & 5.05 & 8.58 & 0.04 \\
\hline \multirow{3}{*}{ With GWO } & MKNN & 3 & 4.18 & 0.03 \\
\cline { 2 - 5 } & Decision tree & 3.57 & 5.25 & 0.14 \\
\cline { 2 - 5 } & Neural Network & 25.68 & 3.80 & 0.11 \\
\cline { 2 - 5 } & Random forest & 1.54 & $\mathbf{3 . 7 4}$ & 0.11 \\
\hline
\end{tabular}

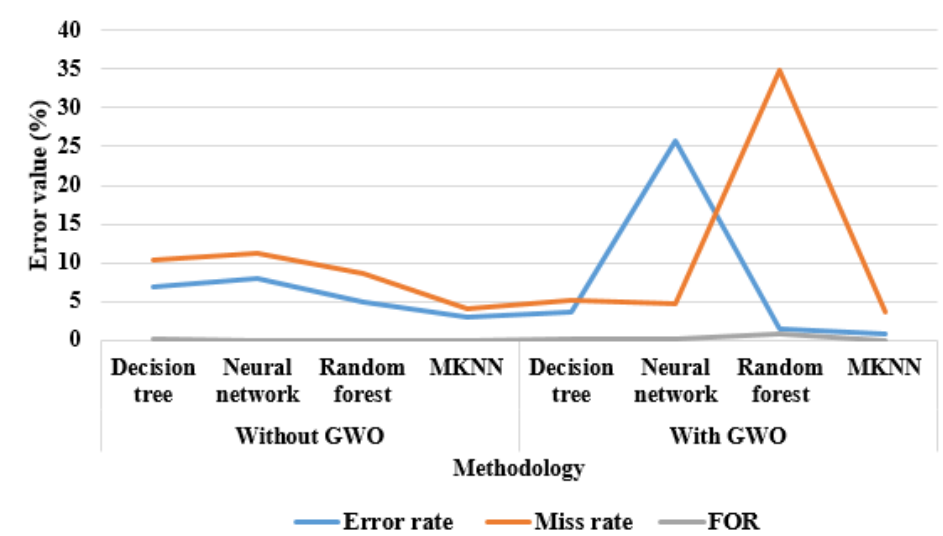

Figure. 6 Graphical comparison of GWO-MKNN model on e-ophtha dataset in terms of miss-rate, error-rate, and FOR

Table 3. Performance evaluation of GWO-MKNN model on DiaRetDB1dataset in light of sensitivity, specificity and accuracy

\begin{tabular}{|c|c|c|c|c|}
\hline \multicolumn{5}{|c|}{ DiaRetDB1 dataset } \\
\hline \multirow{2}{*}{ Optimization } & Classifier & Accuracy (\%) & Sensitivity (\%) & Specificity (\%) \\
\hline \multirow{3}{*}{ Without GWO } & Decision tree & 97.75 & 81.95 & 98.91 \\
\cline { 2 - 5 } & Neural network & 84.62 & 97.13 & 84.51 \\
\cline { 2 - 5 } & Random forest & 97.63 & 72.79 & 97.87 \\
\cline { 2 - 5 } & MKNN & 98.95 & 94.08 & 98.26 \\
\hline \multirow{3}{*}{ With GWO } & Decision tree & 97.98 & 96.40 & 98.99 \\
\cline { 2 - 5 } & Neural network & 68.76 & 98.39 & 67.98 \\
\cline { 2 - 5 } & Random forest & 98.50 & 67.62 & 98.31 \\
\cline { 2 - 5 } & MKNN & $\mathbf{9 9 . 9 0}$ & $\mathbf{9 9 . 0 3}$ & $\mathbf{9 9 . 2 1}$ \\
\hline
\end{tabular}

in classification accuracy. Related to the existing classifiers (decision tree, neural network, and random forest), the MKNN classifier works well in higher dimensional data and easy to implement for multiclass issues. The graphical depiction of the GWOMKNN model on DiaRetDB1dataset in light of sensitivity, specificity and accuracy is stated in Fig. 7.

In Table 4, the proposed GWO-MKNN model performance is investigated using miss rate, error rate, and FOR. In the DiaRetDB1 dataset, the GWO-
MKNN model achieved a minimum error rate of $0.10 \%$, a miss rate of $1.27 \%$ and FOR of $0.01 \%$. In this research study, optimization plays a crucial role in microaneurysms recognition, where GWO significantly selects the microaneurysms regions from the non-microaneurysms regions. The graphical comparison of GWO-MKNN model on DiaRetDB1dataset in terms of miss-rate, error-rate, and FOR is denoted in Fig. 8. 


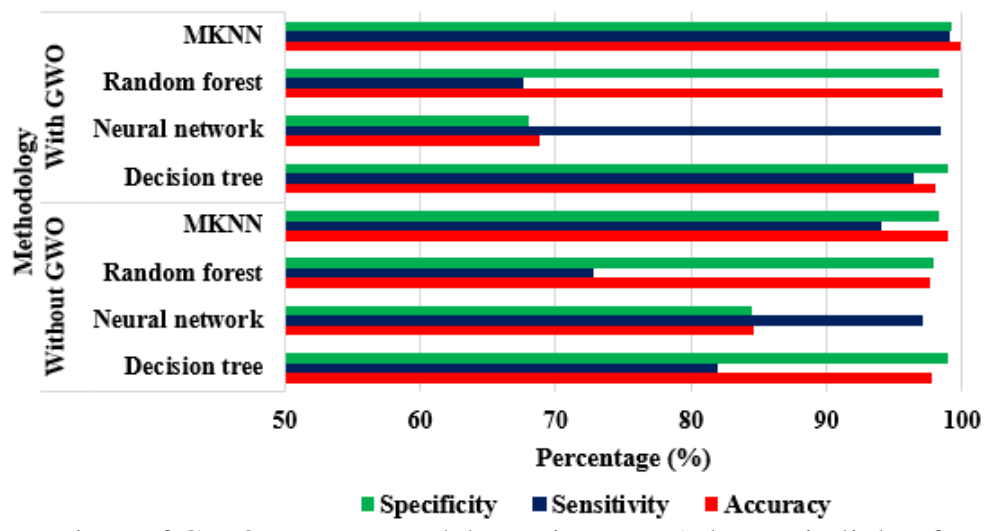

Figure. 7 Graphical comparison of GWO-MKNN model on DiaRetDB1 dataset in light of sensitivity, accuracy, and specificity

Table 4. Performance evaluation of GWO-MKNN model on DiaRetDB1dataset in terms of miss rate, error rate, and FOR

\begin{tabular}{|c|c|c|c|c|}
\hline \multirow{2}{*}{ Optimization } & Classifier & Error rate (\%) & Miss rate (\%) & FOR (\%) \\
\hline \multirow{3}{*}{ Without GWO } & Decision tree & 2.25 & 18.05 & 0.16 \\
\cline { 2 - 5 } & Neural network & 15.38 & 2.87 & 0.09 \\
\cline { 2 - 5 } & Random forest & 2.37 & 27.21 & 0.24 \\
\cline { 2 - 5 } & MKNN & 1.05 & 5.92 & 0.05 \\
\hline \multirow{3}{*}{ With GWO } & Decision tree & 2.02 & 3.60 & 0.10 \\
\cline { 2 - 5 } & Neural network & 31.24 & 1.61 & 0.43 \\
\cline { 2 - 5 } & Random forest & 1.50 & 32.38 & 0.85 \\
\cline { 2 - 5 } & MKNN & $\mathbf{0 . 1 0}$ & $\mathbf{1 . 2 7}$ & $\mathbf{0 . 0 1}$ \\
\hline
\end{tabular}

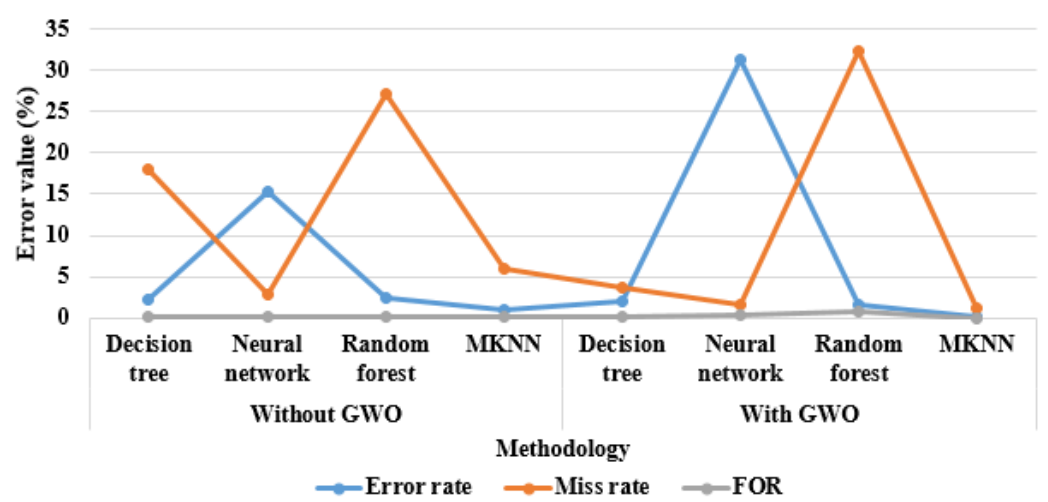

Figure. 8 Graphical comparison of GWO-MKNN model on DiaRetDB1 dataset in terms of miss-rate, error-rate, and FOR

\subsection{Comparative performance}

Comparative performance between the proposed and the existing models is given in table 5. Mazlan [11] introduced a framework for microaneurysms detection in the fundus retinal images. At first, contrast enhancement was used for improving the visibility level of the collected images. Then, $\mathrm{H}-$ maxima and multilevel thresholding methodologies were utilized to decrease the image intensity level and to segment the microaneurysms regions. Around 15 features (major axis length, aspect ratio, eccentricity, mean, perimeter, variance, minor axis length, standard deviation, area, entropy, circularity, etc.) were employed for extracting the features from the segmented microaneurysms regions. The obtained feature vectors were fed to SVM and MLP to classify the retinal images into three classes like background, blood vessels and microaneurysms. In this experimental section, the developed framework attained $92.28 \%$ of accuracy on e-ophtha dataset. Manjaramkar, and Kokare, [16] developed a new 
Table 5. Comparative performance between the proposed and existing models

\begin{tabular}{|c|c|c|}
\hline Methodology & Dataset & $\begin{array}{c}\text { Accuracy } \\
(\mathbf{\%})\end{array}$ \\
\hline $\begin{array}{c}\text { H-maxima-multilevel } \\
\text { thresholding-MLP [11] }\end{array}$ & e-ophtha & 92.28 \\
\hline $\begin{array}{c}\text { Statistical geometrical } \\
\text { features [16] }\end{array}$ & DiaRetDB1 & 84.15 \\
\hline MResUNet [18] & DiaRetDB1 & 99.87 \\
\hline GWO-MKNN model & e-ophtha & $\mathbf{9 9 . 1 0}$ \\
\cline { 2 - 3 } & DiaRetDB1 & $\mathbf{9 9 . 9 0}$ \\
\hline
\end{tabular}

model to detect the microaneurysms in fundus retinal images. In this literature, a set of features were developed based on the statistics of geometrical properties of connected regions for discriminating non-lesion and lesion pixels. In this work, the presented model performance was evaluated on the DiaRetDB1 dataset that obtained $84.15 \%$ of accuracy. Similarly, Qomariah [18] implemented a Modified Residuals UNet (MResUNet) model for an effective microaneurysms classification. As seen in the resulting phase, the developed MResUNet model obtained $99.87 \%$ of accuracy on DiaRetDB1 dataset. Compared to these existing studies, the GWOMKNN model achieved $99.10 \%$ and $99.90 \%$ of classification accuracy on e-ophtha and DiaRetDB1 datasets that showed better improvement in microaneurysms detection.

\section{Conclusion}

In this research paper, the GWO-MKNN model is developed for detecting microaneurysms regions from the fundus images for early detection of DR. The main objective of this work is to propose proper segmentation and classification methodologies for microaneurysms detection. After enhancing the quality of raw retinal images, hessian-based filter and Otsu thresholding with the morphological operator are used to segment and separate the microaneurysms regions and non- microaneurysms regions. For predicting the correctness of microaneurysms regions, the GWO algorithm is applied in this research. Then, the feature vectors are extracted from microaneurysms regions using GLCM and shape features, where the obtained feature vectors are fed to MKNN to classify both microaneurysms and nonmicroaneurysms regions. Related to the existing models (H-maxima-multilevel thresholding-MLP and statistical geometrical features), the GWOMKNN model achieved good performance in microaneurysms detection in light of classification accuracy. The GWO-MKNN model achieved $99.10 \%$ and $99.90 \%$ of classification accuracy on both e-ophtha and DiaRetDB1 datasets that are better related to the prior models. As a future extension, a clustering-based segmentation algorithm is added in GWO-MKNN model to further improve the performance of microaneurysms detection.

\section{Conflicts of interest}

The authors declare no conflict of interest.

\section{Author contributions}

The paper conceptualization, methodology, software, validation, formal analysis, investigation, resources, data curation, writing - original draft preparation, writing-review and editing, visualization, have been done by $1^{\text {st }}$ author. The supervision and project administration, have been done by $2^{\text {nd }}$ author.

\section{References}

[1] P. R. Wankhede and K. B. Khanchandani, "Automated Microaneurysms Detection from Retinal Fundus Images using Pixel Intensity Rank Transform”, Biomedical \& Pharmacology Journal, Vol. 13, No. 1, pp. 47-54, 2020.

[2] P. Chudzik, S. Majumdar, F. Calivá, B. A. Diri, and A. Hunter, "Microaneurysm detection using fully convolutional neural networks", Computer Methods and Programs in Biomedicine, Vol. 158, pp. 185-192, 2018.

[3] S. Jiménez, P. Alemany, F. J. Núñez, I. Fondón, C. Serrano, B. Acha, and I. Failde, "Automated detection of microaneurysms by using region growing and fuzzy artmap neural network", Archivos de la Sociedad Española de Oftalmología, Vol. 87, No. 9, pp. 284-289, 2012.

[4] S. S. Rahim, C. Jayne, V. Palade, and J. Shuttleworth, "Automatic detection of microaneurysms in colour fundus images for diabetic retinopathy screening", Neural Computing and Applications, Vol. 27, No. 5, pp. 1149-1164, 2016.

[5] R. Karthikeyan and P. Alli, "Feature selection and parameters optimization of support vector machines based on hybrid glowworm swarm optimization for classification of diabetic retinopathy", Journal of Medical Systems, Vol. 42, No. 10, p. 195, 2018.

[6] N. Eftekhari, H. R. Pourreza, M. Masoudi, K. G. Shirazi, and E. Saeedi, "Microaneurysm detection in fundus images using a two-step convolutional neural network", Biomedical Engineering Online, Vol. 18, No. 1, p. 67, 2019.

[7] X. Zhang, J. Wu, M. Meng, Y. Sun, and W. Sun, "Feature-transfer network and local background 
suppression for microaneurysm detection", Machine Vision and Applications, Vol. 32, No. 1, pp. 1-13, 2021.

[8] W. Zhou, C. Wu, D. Chen, Y. Yi, and W. Du, "Automatic microaneurysm detection using the sparse principal component analysis-based unsupervised classification method", IEEE Access, Vol. 5, pp. 2563-2572, 2017.

[9] W. Cao, N. Czarnek, J. Shan, and L. Li, "Microaneurysm detection using principal component analysis and machine learning methods", IEEE Transactions on Nanobioscience, Vol. 17, No. 3, pp. 191-198, 2018.

[10] D. J. Derwin, S. T. Selvi, and O. J. Singh, "Discrimination of microaneurysm in color retinal images using texture descriptors", Signal, Image and Video Processing, Vol. 14, No. 2, pp. 369-376, 2020.

[11] N. Mazlan, H. Yazid, H. Arof, and H. M. Isa, "Automated Microaneurysms Detection and Classification using Multilevel Thresholding and Multilayer Perceptron", Journal of Medical and Biological Engineering, pp. 1-15, 2020.

[12] D. Veiga, N. Martins, M. Ferreira, and J. Monteiro, "Automatic microaneurysm detection using laws texture masks and support vector machines", Computer Methods in Biomechanics and Biomedical Engineering: Imaging \& Visualization, Vol. 4, pp. 405-416, 2018.

[13] M. U. Akram, S. Khalid, and S. A. Khan, "Identification and classification of microaneurysms for early detection of diabetic retinopathy", Pattern Recognition, Vol. 46, No. 1, pp. 107-116, 2013.

[14] D. J. Derwin, S. T. Selvi, O. J. Singh, and B. P. Shan, "A novel automated system of discriminating Microaneurysms in fundus images", Biomedical Signal Processing and Control, Vol. 58, p. 101839, 2020.

[15] S. Long, J. Chen, A. Hu, H. Liu, Z. Chen, and D. Zheng, "Microaneurysms detection in color fundus images using machine learning based on directional local contrast", BioMedical Engineering OnLine, Vol. 19, pp. 1-23, 2020.

[16] A. Manjaramkar and M. Kokare, "Statistical geometrical features for microaneurysm detection", Journal of Digital Imaging, Vol. 31, No. 2, pp. 224-234, 2018.

[17] R. E. Putra, H. Tjandrasa, and N. Suciati, "Severity classification of non-proliferative diabetic retinopathy using convolutional support vector machine", International Journal of Intelligent Engineering and Systems, Vol. 13, No. 4, pp. 156-170, 2020.
[18] D. U. N. Qomariah, H. Tjandrasa, and C. Fatichah, "Segmentation of Microaneurysms for Early Detection of Diabetic Retinopathy Using MResUNet", International Journal of Intelligent Engineering and Systems, Vol. 14, No. 3, pp. 359-373, 2021.

[19] E. Decencière, G. Cazuguel, X. Zhang, G. Thibault, J. C. Klein, F. Meyer, B. Marcotegui, G. Quellec, M. Lamard, R. Danno, and D. Elie, "TeleOphta: Machine learning and image processing methods for teleophthalmology", IRBM, Vol. 34, No. 2, pp. 196-203, 2013.

[20] R. V. J. P. H. Kälviäinen and H. Uusitalo, "DIARETDB1 diabetic retinopathy database and evaluation protocol", Medical Image Understanding and Analysis, Citeseer, Vol. 2007, pp. 61, 2007.

[21] X. Dong, G. Wang, Y. Pang, W. Li, J. Wen, W. Meng, and Y. Lu, "Fast efficient algorithm for enhancement of low lighting video", In: Proc. of IEEE International Conference on Multimedia and Expo, Barcelona, Spain, pp. 1-6, 2011.

[22] C. C. R. Aldasoro, "Retrospective shading correction algorithm based on signal envelope estimation", Electronics Letters, Vol. 45, No. 9, pp. 454-456, 2009.

[23] S. K. PS and V. S. Dharun, "Extraction of texture features using GLCM and shape features using connected regions", International Journal of Engineering and Technology, Vol. 8, No. 6, pp. 2926-2930, 2016.

[24] N. Ali, D. Neagu, and P. Trundle, "Evaluation of $\mathrm{k}$-nearest neighbour classifier performance for heterogeneous data sets", SN Applied Sciences, Vol. 1, No. 12, p. 1559, 2019. 